\title{
La théorie de la transposition didactique: un outil conceptuel pour décrire les savoirs grammaticaux élaborés en classe par les élèves
}

\author{
Isabelle Gauvin, Université du Québec à Montréal, Montréal, Canada \\ Marie-Claude Boivin, Université de Montréal, Montréal, Canada
}

(Manuscrit reçu le 15 février 2012, version finale reçue le 15 août, 2012)

\begin{abstract}
Nos travaux ${ }^{1}$ visent à décrire les connaissances grammaticales élaborées par des élèves au cours de l'enseignement/apprentissage de l'accord du verbe. Cette description est réalisée grâce à l'analyse des interactions didactiques, c'est-à-dire les interactions qu'entretiennent les trois pôles du triangle didactique (élève, enseignant et savoir) dans la construction des objets enseignés. La théorie de la transposition didactique de Chevallard (1985/1991) constitue la pierre angulaire de nos travaux. Les concepts de transposition didactique externe (savoir à enseigner) et interne (savoir effectivement enseigné) agissent à titre d'analyseur des interactions didactiques ; le doublet chronogenèse / topogenèse est l'outil conceptuel que nous convoquons pour dépeindre le passage des savoirs à enseigner aux savoirs effectivement enseignés et appris. Cet article mettra l'accent sur les aspects conceptuels et méthodologiques associés à l'étude des interactions didactiques en classe; des résultats seront présentés dans le seul but d’illustrer notre démarche de recherche qui s’inscrit dans le cadre de la théorie de la transposition didactique.
\end{abstract}

\section{Introduction}

Notre recherche ${ }^{2}$ vise la description des connaissances grammaticales élaborées par des élèves québécois de première secondaire (12 ans) au moment de l'enseignement/apprentissage (désormais E/A) de l'accord du verbe (Gauvin, 2011) en classe de français langue première. Plus précisément, l'analyse des interactions didactiques, c'est-à-dire l’interrelation entre les trois pôles du triangle didactique (élève, enseignant et objet de savoir), nous permet de dresser un portrait des connaissances grammaticales que les élèves mobilisent en cours d'E/A. Cet article mettra l'accent sur les aspects conceptuels et méthodologiques associés à l'étude des interactions didactiques en classe ; des résultats seront présentés dans le seul but d'illustrer notre démarche de recherche ainsi que l'opérationnalisation que nous proposons de certains concepts qui sont associés à la théorie de la transposition didactique ${ }^{3}$.

Dans un premier temps, nous situerons nos travaux au sein de la discipline scientifique qu'est la didactique du français. Nous exposerons ensuite le cadre conceptuel qui sert de fondement à nos travaux et montrerons sa pertinence théorique pour l'analyse des interactions didactiques en classe en présentant succinctement quelques résultats issus de notre recherche. 
Nous verrons, en conclusion, comment nos travaux contribuent à raffiner certains concepts clés des théories didactiques.

\section{Notre recherche au sein de la discipline « didactique du français »}

Le didacticien place au cœur de ses préoccupations les savoirs disciplinaires dans leur interaction avec l'enseignant et les élèves. Dans le cas de la didactique du français langue première, ces savoirs disciplinaires sont notamment ceux transposés à partir des savoirs des disciplines contributoires reliées à l'étude de la langue, soit la linguistique et la littérature. Comme les autres didactiques, la didactique de la langue est à la croisée d'une deuxième famille de disciplines qu'on peut qualifier de contributoires: il s’agit des différentes disciplines qui portent sur l'étude des phénomènes d'enseignement et d'apprentissage, comme la pédagogie, à la psychopédagogie ou encore à la psychologie (Simard, Dufays, Dolz, \& Garcia-Debanc, 2010).

Dans notre recherche qui cherche à décrire comment les élèves élaborent, en classe de français, les savoirs relatifs à l'accord du verbe ${ }^{4}$, les savoirs grammaticaux sont cruciaux. Toutefois, ce ne sont pas les savoirs issus de la linguistique qui en sont le principal fondement, mais plutôt ceux qui ont fait l'objet de transposition pour l'E/A. Ainsi, nous nous intéressons à la manière dont les élèves élaborent leurs connaissances au sujet de l'accord du verbe, tel que cet objet est défini en grammaire pédagogique moderne (Boivin \& Pinsonneault, 2008). Notre travail porte donc sur l'analyse des interactions didactiques entre les élèves (en priorité) et l'enseignant (dans une moindre mesure) relativement à cet objet de savoir. Notre analyse des interactions didactiques repose sur l'étude des interactions verbales entre élèves d'une part, et entre les élèves et leur enseignant d'autre part, au moment de travail individuel, de travail en dyades et de discussion avec l'ensemble de la classe.

Nos données, c’est-à-dire les interactions verbales, ont été collectées dans dans sept classes de français langue première de $1^{\text {re }}$ secondaire (12 ans) au Québec. Nous avons constitué un corpus de films de classe de 22 leçons portant sur l'accord du verbe. Les films de classe ont été transcrits sous forme de verbatims afin d'être analysés selon deux perspectives : macro et micro. Seuls quelques résultats de l'analyse micro seront présentés dans cet article, et ce, afin d'illustrer notamment comment l'opérationnalisation que nous proposons de certains concepts a pu servir notre analyse.

Quiconque s’intéresse à l'élaboration des savoirs par les élèves convoquerait sans doute plus spontanément un cadre de référence lié à la deuxième famille de disciplines contributoires à 
la didactique, celle qui concerne l'étude des phénomènes d'enseignement et d'apprentissage. Il est vrai que les divers concepts associés aux théories de l'apprentissage (nous pensons plus particulièrement aux théories socioconstructiviste, constructive et cognitiviste) peuvent agir à titre d'analyseur des interactions entre les trois pôles du triangle didactique. Nous avons toutefois choisi d'appuyer délibérément notre analyse des interactions didactiques sur un cadre conceptuel qui s'inscrit directement dans la didactique des disciplines.

\section{Choix d'un cadre de référence didactique}

Nous souhaitions en effet adopter une posture qui s'inscrit directement dans le champ de la didactique par les cadres théoriques didactiques convoqués. Selon nous, la description et la théorisation des interactions didactiques peuvent désormais, en l'état actuel de l'avancement de la discipline qu'est la didactique, s'appuyer sur un réseau de concepts propres à cette discipline. Pourtant, tout un pan de la recherche en didactique s'intéresse à l'E/A en classe, sans toutefois s'appuyer sur les concepts qui ont émergé de la discipline. En fait, la plupart de ces recherches ont adopté d'autres perspectives théoriques que celle que nous privilégions. Moro et Rickenmann (2004) en recensent plusieurs : le pragmatisme, l'interactionnisme symbolique, le paradigme de la cognition située, le paradigme ethnométhodologique et conversationnel et le paradigme historico-culturel vygotskien. Ces diverses perspectives, loin d'être inappropriées ou inutiles, font appel à un ensemble de concepts pour interpréter les situations d'E/A en classe, concepts qui relèvent rarement exclusivement de la didactique ; pour cette raison, nous avons fait le choix de ne pas les retenir pour notre recherche.

Les quelques rares recherches qui s'intéressent aux interactions didactiques dans une perspective exclusivement didactique prennent assise sur deux théories complémentaires, théories qui agissent à titre d'instruments conceptuels pour la description de la dynamique des interactions didactiques (Canelas-Trevis, 2009; Halté, 1998): la théorie de la transposition didactique de Chevallard (1985/1991) et la théorie des situations didactiques de Brousseau (1998). Ces théories, développées en didactique des mathématiques, commencent à s'implanter en didactique du français.

Les contributions scientifiques de Chevallard (1985/1991) et de Brousseau (1998) sont riches en concepts qui permettent d'opérationnaliser leur théorie : par exemple, les concepts de « texte du savoir » et de « temps didactique » chez Chevallard contribuent à rendre opératoire sa théorie de la transposition didactique. Il en va de même des concepts de «dévolution » et de 
« contre-dévolution » ou de « milieu » chez Brousseau. L'ensemble du réseau conceptuel issu de ces théories peut s'avérer pertinent pour la description et la théorisation des interactions didactiques en classe. Cependant, puisqu'il nous apparaissait impossible dans le cadre d'une thèse d'appuyer notre recherche sur l'ensemble du réseau de concepts, nous avons choisi de faire appel à seulement quatre concepts clés les plus fréquemment convoqués en didactique du français : les concepts de transposition didactique interne, de chronogenèse, de topogenèse et d'institutionnalisation (ce dernier concept ne fera pas l'objet de discussion ici). Nous croyons que nos travaux contribuent à montrer la pertinence théorique et la robustesse de ces concepts les plus souvent cités et, par conséquent, à alimenter ces cadres théoriques pour la discipline « français » : nous y reviendrons en conclusion.

\section{Un cadre conceptuel didactique}

La théorie de la transposition didactique de Chevallard (1985/1991) constitue la pierre angulaire de nos travaux. Après avoir défini la tranposition didactique interne, nous la lierons aux concepts de chronogenèse et de topogenèse. Nous verrons enfin en quoi la transposition didactique interne éclaire la transposition didactique externe.

La transposition didactique interne comme analyseur des interactions didactiques

Les contenus de savoir «savant» ou scientifique destinés à devenir des contenus à enseigner exigent nécessairement une adaptation en contenus d'enseignement: ce processus de transformation, la transposition didactique, a fait l'objet d'une théorisation par Chevallard $(1985 / 1991)$ :

Un contenu de savoir ayant été désigné comme savoir à enseigner subit dès lors un ensemble de transformations adaptatives qui vont le rendre apte à prendre sa place parmi les objets d'enseignement. Le « travail » qui d'un objet de savoir à enseigner fait un objet d'enseignement est appelé la transposition didactique. (p. 39)

Ainsi, les savoirs, reconnus comme tels socialement et culturellement, sont ceux qui se voient accorder une légitimité pour l'enseignement. Mais ils ne peuvent être enseignés tels quels : afin d'être enseignables, ils doivent être transformés. Loin d'être une simple vulgarisation du savoir «savant », le savoir transposé est une création originale. Dans le contexte scolaire, les savoirs transformés et adaptés par la transposition didactique externe se retrouvent explicitement dans les programmes d'études, le matériel didactique, etc. (Chevallard, 1985/1991); dans le cas de la grammaire, ces savoirs se retrouvent également dans les grammaires pédagogiques. 
Il arrive parfois que le savoir effectivement enseigné diffère du savoir à enseigner. Les mécanismes par lesquels un objet à enseigner se transforme en un objet réellement enseigné relèvent de la transposition didactique interne (Chevallard, 1985/1991). Ainsi, le «mouvement transpositionnel » (Bronckart \& Plazaola-Giger, 1998) se concrétise en plusieurs étapes, sur plusieurs niveaux : des contenus de savoir aux contenus à enseigner, des contenus à enseigner aux contenus effectivement enseignés, et, pour certains auteurs, des contenus effectivement enseignés aux contenus appris (Raisky \& Caillot, 1996).

Pour le chercheur, la description et l'analyse des objets effectivement enseignés, c'est-àdire la description et l'analyse de la transposition didactique interne, peuvent être abordées du point de vue de l'enseignant qui présente les savoirs à l'élève ou de celui de l'élève qui se les approprie (Schneuwly \& Thévenaz-Christen, 2006). Si on cherche à décrire la TD interne en se centrant sur l'élève, les objets transposés sont dévoilés, notamment, par les interventions des élèves qui tentent de se les approprier, par leurs travaux, par leurs connaissances effectivement construites, par les connaissances qu'ils mobilisent: ce type de recherche se centre sur la transformation de l'objet enseigné en analysant le travail de l'élève (Schneuwly \& ThévenazChristen, 2006).

En didactique du français, la plupart des travaux qui se sont intéressés à la transposition didactique interne se sont attardés au pôle « enseignant» du triangle didactique, comme le soulignent Schneuwly et Thévenaz-Christen (2006) et Boivin (2009). Notre recherche se propose de porter une attention particulière au pôle "élève », puisque notre objectif général de recherche est de décrire comment les élèves élaborent les savoirs relatifs à l'accord du verbe au moment de son E/A. Si le concept de transposition didactique interne nous apparait particulièrement productif pour décrire l'élaboration du savoir grammatical des élèves en classe autour de cette notion, il doit cependant faire l'objet d’une opérationnalisation pour nos travaux de recherche.

Nous posons comme prémisse que les transformations que les élèves font subir aux savoirs en cours d'E/A permettent d'inférer comment ils élaborent leurs connaissances. Dans notre recherche, les transformations des savoirs portent évidemment sur la règle d'accord du verbe, mais également sur l'identification du verbe, l'identification du sujet et le transfert des traits de personne et de nombre telles que nous avons défini ces objets a priori ${ }^{5}$. Les transformations des savoirs s'observent à partir des interventions ${ }^{6}$ des élèves en classe, alors que ces derniers tentent de s’approprier ces objets grammaticaux. Les interventions sont constituées 
plus précisément des questions qu’ils posent, des réponses qu’ils fournissent, des commentaires métagraphiques qu'ils produisent, des hypothèses qu'ils formulent, etc. L’écart, plus ou moins important, ou l'absence d'écart entre les savoirs à enseigner, tel que défini a priori, et les savoirs tels qu'ils s’actualisent dans les interventions des élèves permettent de décrire les transformations que les élèves font subir, en cours d'apprentissage, aux objets de savoirs (Schneuwly, Sales Cordeiro, \& Dolz, 2005) ou, pour dire autrement, permettent de décrire la transposition didactique interne.

\section{Chronogenèse et topogenèse: outils conceptuels pour décrire la transposition didactique interne}

Les deux concepts frères de chronogenèse et de topogenèse agissent à titre de filtres pour l'analyse des interactions didactiques et permettent de comprendre la transposition didactique interne. Pour Chevallard (1885/1991), la chronogenèse concerne l'avancement des savoirs dans le temps :

Dans la relation didactique (qui unit enseignant, enseignés et « savoirs »), l'enseignant est le servant de la machine didactique dont le moteur est la contradiction de l'ancien et du nouveau : il en nourrit le fonctionnement en y introduisant ces objets transactionnels que sont les objets de savoir convenablement apprêtés en objets d'enseignement. (Chevallard, 1985/1991, p. 71) ${ }^{7}$.

Ainsi, l'enseignant mène la chronogenèse : dans l'interaction didactique classique, l'enseignant est considéré comme le principal responsable de l'avancement des savoirs en classe (Chevallard, 1985/1991). Il anticipe et dirige la chronogenèse, alors que les élèves ne le peuvent généralement pas: Sensevy (1998) dira que l'enseignant est «chronomaitre ». Toutefois, il importe de reconnaitre que des élèves partagent parfois cette responsabilité : ces élèves sont alors appelés « élèves chronogènes » (Boivin, 2007; Sensevy, 1998). Les élèves chronogènes se distinguent par les percées dans l'avancement du savoir en construction auxquelles ils contribuent significativement.

[...] certains élèves, dont les interventions, les questions ou les remarques font avancer le savoir ou le présentent autrement, peuvent avoir une fonction chronogène dans la classe (Schubauer-Leoni \& Leutenegger, 2002, Sensevy, 1998). Ces élèves ne “conduisent” bien entendu pas l'avancement des savoirs, mais ils y participent, ils y contribuent. En d'autres termes, du point de vue didactique, on ne peut ignorer l'apport de tels élèves si on veut véritablement comprendre le fonctionnement de la chronogenèse. » (Boivin, 2007, p. 292) 
L'enseignant est donc principalement celui qui, à l'égard du savoir en construction, « sait avant »; il est également celui qui « sait autrement » (Chevallard, 1985/1991). D’un autre point de vue, les positions de l'enseignant et des élèves à l'égard du savoir en construction sont différentes, et les places respectives qu'ils occupent par rapport au savoir définissent la topogenèse. Plus précisément, la topogenèse porte sur le partage des tâches et des responsabilités entre les acteurs de l'interaction didactique, un partage qui, pour Chevallard (1985/1991), semble aller de soi : l'enseignant est celui qui détient un certain savoir, l'élève, qui en détient un autre, est celui qui doit se l'approprier. La topogenèse est liée à la chronogenèse. En effet, les positions de chacun imposent des contraintes, des conditions topogénétiques (Boivin, 2007), quant aux rôles de l'enseignant et de l'élève à l'égard de la chronogenèse, à l'égard de la construction du savoir dans le temps. Des actions comme démontrer, généraliser, appliquer, interroger, répondre, etc. ne sont que quelques exemples de rôles qui incombent aux acteurs de l'interaction didactique (Boivin, 2007; Chevallard, 1985/1991; Sensevy, 1998).

Opérationnalisation des concepts de chronogenèse et topogenèse pour notre recherche Pour Chevallard (1985/1991), l'enseignant, parce qu'il sait avant, conduit l'avancement des savoirs dans le temps : cette conception laisse présumer que le savoir se projette vers l'avant, sans faire de sur-place ou de retours en arrière. Or, notre recherche portant sur l'E/A de l'accord du verbe suggère qu'une véritable compréhension de la chronogenèse demande de s'intéresser aux avancées, mais également aux piétinements et aux reculs dans la construction des savoirs au cours de la transposition didactique interne. Le choix des termes « piétinement » et « recul » peut donc paraitre paradoxal si la définition de la chronogenèse se limite à l'« avancement du savoir ». Pour cette raison, nous préférons définir la chronogenèse comme l'évolution des savoirs dans le temps. À nos yeux, l'absence d'évolution reflète un état, l'état 0 , par rapport à un avancement qui reflèterait l'état +1 . Au même titre, un recul, qui correspondrait à état -1 , constitue un état de l'évolution du savoir. À ce stade-ci, il s'agit que d'hypothèses qui justifieront certains aspects méthodologiques: nous reviendrons, en conclusion, sur la pertinence de ces hypothèses.

Dans notre recherche, qui s’intéresse prioritairement au travail de l'élève, la chronogenèse peut donc être appréciée à travers les avancées, les piétinements ou les reculs que les élèves font subir à l'évolution des connaissances relatives à l'accord du verbe, aux transformations qu'ils font subir aux objets « identification du verbe », « identification du sujet », « transfert des traits 
de personne et de nombre » et « règle d'accord ». Ces avancées, piétinements et reculs peuvent être révélés par certaines interventions des élèves, puisque des questions, des réponses, des commentaires métagraphiques, etc. contribuent à l'évolution dans la construction des savoirs en classe, à la transformation du savoir en classe. Ainsi, l'observation de l'évolution des savoirs dans le temps porte sur ce que nous avons choisi d'appeler les interventions chronogènes: les interventions chronogènes agissent donc à titre de révélateur de l’appropriation par les élèves de certains aspects du savoir.

Nous pensons qu'il est possible d’évaluer la position et le rôle des acteurs de l'interaction didactique grâce à l'observation des interventions chronogènes. Alors que l'enseignant détient un certain savoir autour de l'accord du verbe (le savoir à enseigner), nous supposons que les élèves en détiennent un autre qui contribue à l'évolution des savoirs en classe : nous proposons de nommer ce savoir «savoir-en-évolution ». Le savoir-en-évolution des élèves a la particularité d’être différent du savoir à enseigner et de faire avancer, reculer ou piétiner l'élaboration des savoirs.

Dans notre recherche, les concepts de chronogenèse et topogenèse constituent des outils conceptuels utiles pour décrire le passage des savoirs à enseigner aux savoirs effectivement enseignés. Plus précisément, ces concepts ont servi de filtres pour l'analyse des données, comme nous le verrons avec quelques résultats de notre recherche. Étant donné le but de l'article, qui est de discuter de l'opérationnalisation que nous proposons de certains concepts didactiques dans une recherche en didactique du français langue première, nous ne pourrons reprendre l'ensemble des résultats, publiés ailleurs (Gauvin, 2011; soumis ; Gauvin \& Boivin, 2012, soumis).

\section{Chronogenèse et topogenèse : une illustration}

Dans notre recherche, nous avons sélectionné un certain nombre d’évènements remarquables (Boivin, 2007; Schubauer-Leoni \& Leutenegger, 2002) sur la base de critères chronogénétiques : plus précisément, des évènements remarquables ont été choisis parce que les interventions des élèves marquent une avancée, un piétinement ou un recul dans l’évolution des savoirs en classe.

Prenons un exemple d’évènement remarquable sélectionné parce que les interventions des élèves font reculer les savoirs : leurs interventions les conduisent à s’éloigner du savoir à enseigner, tel que défini a priori. Dans une classe, les élèves doivent identifier les verbes dans des phrases décontextualisées, dont celle-ci: À l'envoi de billets au XIXe siècle a succédé 
l'échange de cartes de vœux. L’échange suivant a été recueilli au moment de la correction par l'enseignant de l'exercice avec l'ensemble de la classe.

Phrase : À l'envoi de billets au XIXe siècle a succédé l'échange de cartes de vœux.

Élève 1 : Envoi.

(...)

Élève 1 : On peut dire j'envoie, tu m'envoies, il envoie, nous envoyons.

Enseignant : T'as raison. Donc tu dis, en voyant le mot envoi, j'peux dire j'envoie, tu envoies, il envoie.

Élève 2 : On peut dire n'envoie pas.

Les élèves 1 et 2 pensent que le nom envoi est un verbe : pour s'en convaincre, ils utilisent les deux manipulations décisives ${ }^{8}$ pour identifier le verbe, c'est-à-dire la conjugaison (j'envoie, tu m'envoies, il envoie, nous envoyons) et l'ajout du marqueur de négation autour du verbe (n'envoie pas). Il est crucial de noter que, dans cette interaction, ces manipulations syntaxiques sont appliquées hors phrase; si elles avaient été utilisées dans la phrase (*À l'envoyait de billets... *À le n'envoie pas de billet... ${ }^{9}$ ), les élèves n'auraient eu d'autre choix que de rejeter la nouvelle construction et de conclure, par le fait même, que envoi n’est pas un verbe.

Dans nos données, 35 autres évènements de ce type, ce qui correspond à 31\% des évènements remarquables retenus, concernent l'emploi des manipulations hors phrase pour identifier le verbe ou le sujet. Ce résultat est révélateur de l'élaboration des savoirs relatifs à l'accord du verbe, à savoir que l'emploi des manipulations syntaxiques hors phrase pour identifier le verbe ou le sujet empêche généralement le savoir d’avancer.

À l’opposé, 12 évènements remarquables, soit 10\% des évènements remarquables relevés, montrent que le savoir avance lorsque les manipulations syntaxiques sont employées dans le contexte de la phrase, comme l'illustre ce deuxième extrait, où des élèves, lors d'un travail en dyade, tentent d’identifier le sujet dans des phrases décontextualiés.

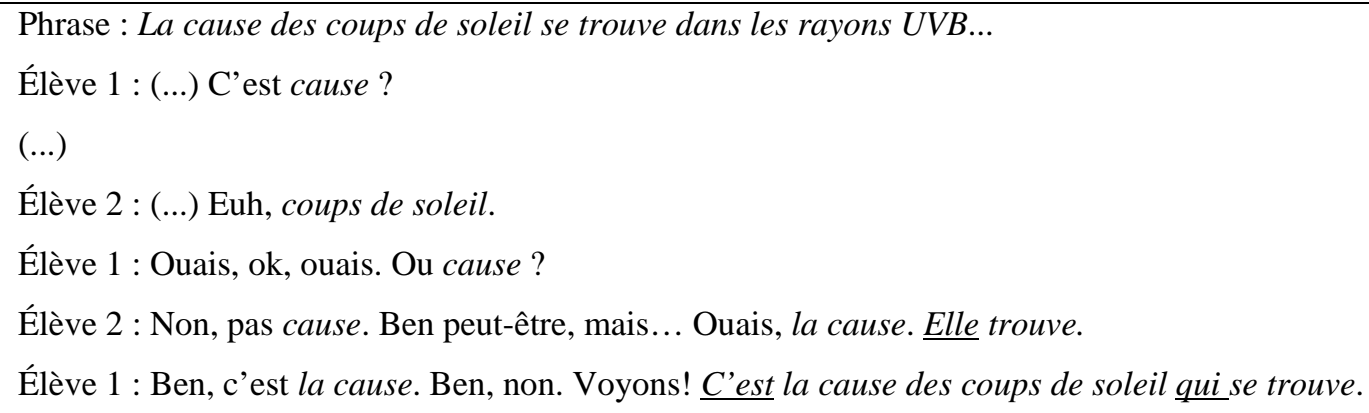

Dans cette phrase, le sujet est la cause des coups de soleil, dont le noyau est cause. Les élèves hésitent: est-ce que le sujet est cause ou coups de soleil ? L’élève 2 effectue le remplacement de 
ce qu'il pense être le sujet (la cause) par un pronom de conjugaison (elle), ce qui tend à confirmer l'hypothèse que la cause est le sujet, parce que c'est également le pronom qui aurait remplacé le sujet complet. Toutefois, si cette manipulation décisive pour identifier le sujet avait été employée dans la phrase (*Elle des coups de soleil se trouve...), l'élève 2 n'aurait pu conclure que la cause est le sujet de la phrase. C'est finalement l'élève 1, à la toute fin, qui propose l'ajout de c'est... qui autour du sujet la cause des coups de soleil et qui réussit, grâce à cette manipulation décisive employée dans la phrase, à faire avancer le savoir.

Ces deux exemples illustrent comment la sélection et l'analyse d'évènements remarquables selon les critères chronogénétiques «avancée », « recul » et «piétinement » permettent d'apprécier la transposition didactique interne et de décrire l'élaboration des savoirs par les élèves. Nous avons supposé que la mise au jour du savoir dont disposent les élèves dans les moments clés de la chronogenèse permet de caractériser leur savoir, savoir qui a la particularité d'être différent du savoir à enseigner et de faire avancer, ou non, la construction des savoirs. L'interaction suivante illustre en quoi cet évènement remarquable, sélectionné sur la base du critère chonogénétique "piétinement », est révélateur du savoir des élèves : le verbe varie en nombre et personne, mais cette procédure nécessite un ajustement dans le cas d'un verbe pronominal (le pronom réfléchi, parce qu'il fait partie du verbe, varie également en nombre et personne).

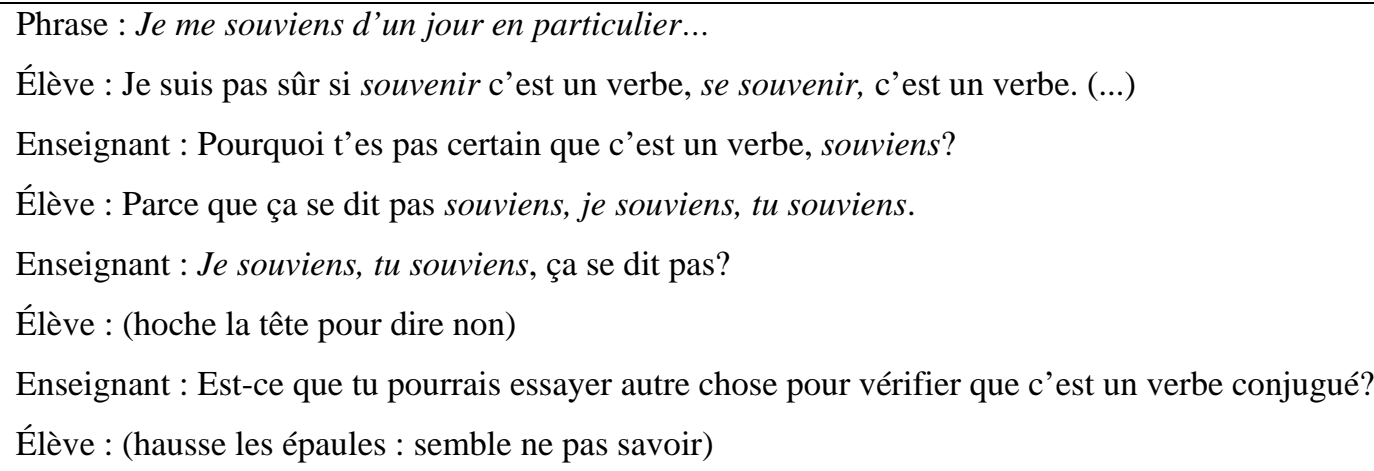

L'élève, qui pose une question à son enseignement au moment où il complète un exercice d'identification du verbe dans des phrases décontextualisées, recourt à la conjugaison pour tenter d'identifier le verbe (*je souviens, *tu souviens), mais ne considère pas le pronom lié au verbe pronominal dans la manipulation: le résultat, *je souviens, *tu souviens, est donc agrammatical, ce qui le fait douter de son statut de verbe. Dans notre corpus de films de classe, 26 évènements remarquables (soit 22\% de tous les évènements remarquables) portent sur la difficulté des élèves 
à adapter les manipulations syntaxiques dans certains contextes comme celui-ci: ce résultat met en lumière l'importance d'inclure, dans le savoir à enseigner, l'enseignement de l'adaptation des manipulations syntaxiques dans ces contextes.

\section{La transposition didactique interne au service de la transposition didactique externe}

La description des connaissances élaborées par les élèves en cours d’E/A, proposée par Gauvin (2011) dans sa thèse, permet non seulement d'éclairer la transposition didactique interne mais également la transposition didactique externe, en ce qui concerne le choix des contenus à enseigner. Sur le plan de la transposition didactique interne, nos résultats montrent, entre autres choses, que l'utilisation ses manipulations syntaxiques à l'extérieur du contexte de la phrase a un effet négatif sur l'évolution des savoirs, tout comme l'absence d'adaptation des manipulations syntaxiques dans certains contextes.

Le portrait détaillé des connaissances élaborées par les élèves que nous avons proposé dans notre recherche (et qui n’a été que très brièvement esquissé ici) nous autorise à reconsidérer les savoirs à enseigner à propos des notions de verbe, de sujet et d'accord du verbe, ainsi que ceux relatifs à l'emploi des outils de la grammaire moderne, c'est-à-dire les manipulations syntaxiques et la phrase de base. À titre d'exemple, il est apparu primordial que le savoir à enseigner conduise les élèves à employer prioritairement et systématiquement les manipulations dans le contexte de la phrase, que ce soit pour identifier le verbe ou le sujet.

Mais la principale conclusion que nous tirons sur le plan de la transposition didactique externe est que le savoir à enseigner est celui qui assure une déconstruction et une reconstruction du savoir élaboré en cours d'E/A. Nous soutenons que l'enseignant a un rôle particulièrement important dans ce processus qui vise à amener les élèves à prendre conscience, en cours d'E/A, des limites du savoir qu'ils utilisent afin de les amener à le transformer, malgré sa grande résistance, en un savoir toujours plus opérationnel. En ce sens, nous inscrivons nos recommandations dans la foulée des travaux de Giordan et ses collaborateurs (Pellaud, Eastes, \& Giordan, 2005). Cette équipe de didacticiens des sciences a développé un «modèle de processus de transformation des connaissances », le modèle allostérique, qui soutient, entre autres choses, qu'il est nécessaire

« [...] de penser la transformation du savoir comme un processus simultané de "déconstruction-reconstruction” [...], où l'ancien savoir serait transformé et ne cèderait 
véritablement la place au nouveau que lorsqu’il apparaît périmé. » (Pellaud et al., 2005, p. 22)

Ainsi, l'enseignement doit faire la démonstration que les connaissances élaborées par les élèves sont périmées ou, pour le dire autrement, restreintes, peu opérationnelles, incomplètes, parfois invalides. Pour transformer le savoir, il importe donc de mettre en place un dispositif d'E/A introduisant une "perturbation cognitive » qui amènera l'élève à prendre conscience que la connaissance élaborée antérieurement n’est pas adéquate, qui l’obligera « à aller plus loin que ce qu’il croit connaître », « à percevoir les limites de son propre raisonnement » (Pellaud, Giordan, \& Eastes, 2007, p. 30). Une fois la connaissance ébranlée, l'apprenant sera plus à même de la transformer en une connaissance mieux adaptée.

\section{Conclusion}

Nous souhaitions mettre à l'épreuve certains concepts clés des théories didactiques, dont ceux de transposition didactique interne, de chronogenèse et de topogenèse. Plus précisément, nous avons tenté de valider empiriquement nos propositions d’opérationnalisation de ces concepts.

Nous proposions de décrire la transposition didactique interne par l'observation de l'écart ou de l'absence d'écart entre le savoir à enseigner, tel que nous l'avons défini au préalable, et le savoir effectivement enseigné, tel qu'il se manifeste dans les interventions des élèves au cours de l’E/A de l'accord du verbe. Nos résultats, dont nous avons très succinctement présenté quelques éléments saillants, nous permettent d'affirmer que les connaissances élaborées par les élèves à propos de l'accord du verbe ne sont pas une copie conforme du savoir à enseigner et du savoir effectivement enseigné. En ce sens, le concept de transposition didactique interne nous apparait tout à fait opérationnel.

Au sujet des concepts de chronogenèse et de topogenèse, nous avions adopté une position a priori: la chronogenèse et la topogenèse peuvent être envisagées du point de vue de l'enseignant, comme le soutient Chevallard (1985/1991), mais aussi du point de vue de l'élève. Cette proposition a par ailleurs été formulée par d'autres avant nous (Boivin, 2007; Sensevy, 1998). Nous suggérions également que l’observation des transformations que les élèves font subir au savoir ne s'effectue pas seulement en examinant les avancées du savoir en classe, mais aussi en examinant les piétinements et les reculs; tenir compte de la chronogenèse dans une recherche portant sur l'E/A de l'accord du verbe demande donc de s'intéresser à la manière dont les savoirs avancent ou n’avancent pas en classe. Nos analyses confirment la pertinence de s'attarder à ces 
trois «temps » dans la description de la transposition didactique interne. En ce sens, notre proposition, qui consistait à envisager la chrononogenèse non pas uniquement en termes d'avancement des savoirs dans le temps (ce qui exclurait implicitement les surplaces et les reculs), mais en termes d'évolution des savoirs dans le temps, trouve tout à fait sa place empiriquement.

Nous avons soutenu que l'évolution des savoirs pouvait être appréciée grâce à ce que nous avons appelé les interventions chronogènes, c’est-à-dire des interventions qui font avancer la construction du savoir, la font reculer ou piétiner. Ainsi, les interventions chronogènes agissent à titre de révélateur de la transposition didactique interne et de l'appropriation, par les élèves, du savoir enseigné. D’autres avant nous, dont Sensevy (1998) et Boivin (2007) ont établi que des interventions d'élèves, nommés élèves chronogènes, contribuent à l'avancement des savoirs dans le temps. Compte tenu de notre définition de la chronogenèse, l'étiquette « chronogène » prend un sens plus large, celui de « qui fait évoluer le savoir ». En conséquence, les élèves visés par Sensevy (1998) et Boivin (2007), ceux qui font avancer les savoirs, pourraient être par exemple être qualifiés d'élèves positivement chronogènes.

Alors que l'enseignant détient un certain savoir autour de l'accord du verbe (le savoir à enseigner) et qu'il sait autrement (Chevallard, 1985/1991), nos analyses des interventions des élèves montrent que ces derniers en détiennent parfois un autre qui contribue à l'évolution des savoirs en classe, un savoir-en-évolution. Ce savoir-en-évolution a la particularité d'être différent du savoir à enseigner et de faire avancer, reculer ou piétiner l'évolution des savoirs en classe. L'examen des avancées, des piétinements et des reculs dans l'évolution des savoirs en classe a permis de caractériser ce savoir des élèves.

Le choix d'un cadre théorique didactique pour étudier l'E/A d'une notion grammaticale s’est avéré productif. Notre description de l'appropriation par les élèves, en classe, des savoirs impliqués dans l'accord du verbe, parce qu'elle repose sur divers concepts didactiques, s’inscrit dans un cadre didactique et contribue à la validation d'un système conceptuel propre à la didactique du français, plus particulièrement pour l'objet «grammaire ». D'autres travaux contribueront sans aucun doute à alimenter ce cadre théorique, relativement récent pour cette discipline. 


\section{Références}

Boivin, M.-C. (2007). Situations didactiques et enseignement de la grammaire: quelques aspects topogénétiques et chronogénétiques. Revue suisse des sciences de l'éducation, 29(2), 279370.

Boivin, M.-C. (2009). Jugements de grammaticalité et manipulations syntaxiques dans le travail en classe d'élèves du secondaire. In J. Dolz \& C. Simard (Eds.), Pratiques d'enseignement grammatical. Points de vue de l'enseignant et de l'élève (pp. 179-208). Québec: Presses de l'Université Laval.

Boivin, M.-C., \& Pinsonneault, R. (2008). La grammaire moderne. Description et éléments pour sa didactique. Montréal: Beauchemin.

Bronckart, J.-P., \& Plazaola-Giger, I. (1998). La transposition didactique en français. Pratiques, 97-98, 35-58.

Brousseau, G. (1998). Théorie des situations didactiques : didactique des mathématiques 1970-1990. Grenoble: La Pensée sauvage.

Canelas-Trevis, S. (2009). La grammaire enseignée en classe. Le sens des objets et des manipulations. Berne: Peter Lang.

Chevallard, Y. (1985/1991). La transposition didactique: du savoir savant au savoir enseigné (2e ed.). Grenoble: Éd. La Pensée sauvage.

Gauvin, I. (2011). Interactions didactiques en classe de français: enseignement/apprentissage de l'accord du verbe en première secondaire. Thèse de doctorat: Université de Montréal.

Thèse disponible à l'adresse suivante: https://papyrus.bib.umontreal.ca/jspui/handle/1866/5281

Gauvin, I. (2012; soumis). Proposition de transposition didactique de la règle d'accord du verbe fondée sur une description des interactions didactiques en classe. Actes du colloque Le complexe du verbe.

Gauvin, I., \& Boivin, M.-C. (2012). Transposition didactique interne et aspects clés de l'apprentissage de l'accord du verbe en français. Revue canadienne de linguistique appliquée, 15(1), 146-166.

Gauvin, I., \& Boivin, M.-C. (2012; soumis). Identifier le verbe dans le but de l'accorder: l'élaboration des connaissances par les élèves en classe. Revue des sciences de l'éducation.

Halté, J.-F. (1998). L'espace didactique et la transposition. Pratiques, 97-98, 177-192.

Moro, C., \& Rickenmann, R. (2004). Situation éducative et significations. Bruxelles: De Boeck.

Pellaud, F., Eastes, R.-E., \& Giordan, A. (2005). Un modèle pour comprendre l'apprendre: le modèle allostérique. Gymnasium Helveticum, 1(5), 18-24.

Pellaud, F., Giordan, A., \& Eastes, R.-E. (2007). Vers de nouveaux paradigmes scolaires. Chemin de traverse, 5, 11-35.

Raisky, C., \& Caillot, M. (1996). Au-delà des didactiques, le didactique: débats autour de concepts fédérateurs. Bruxelles: De Boeck Université.

Schneuwly, B., Sales Cordeiro, G., \& Dolz, J. (2005). À la recherche de l'objet enseigné: une démarche multifocale. Les dossiers des sciences de l'éducation, 14, 77-93.

Schneuwly, B., \& Thévenaz-Christen, T. (2006). Analyse des objets enseignés: le cas du français. Bruxelles: de Boeck.

Schubauer-Leoni, M., \& Leutenegger, F. (2002). Expliquer et comprendre dans une approche clinique/expérimentale du didactique ordinaire. In F. Leutenegger \& M. Saada-Robert (Eds.), Expliquer et comprendre en sciences de l'éducation (pp. 227-251). Bruxelles: De Boeck. 
Sensevy, G. (1998). Institutions didactiques: étude et autonomie à l'école élémentaire. Paris: PUF.

Simard, C., Dufays, J.-L., Dolz, J., \& Garcia-Debanc, C. (2010). Didactique du français langue français. Bruxelles: de Boeck.

${ }^{1}$ Ce texte est accessible sur le site du colloque de l'AFIRSE 2011 (http://www.afirsecongres2011.info/). Il est publié ici presque tel quel afin d'atteindre un plus large public.

${ }^{2}$ Recherche financée grâce à une subvention de recherche du Fonds québécois de recherche sur la société et la culture (subvention 108020 octoyée à Marie-Claude Boivin) et à deux bourses doctorales octroyées à Isabelle Gauvin par le Fonds québécois de recherche sur la société et la culture de 2005 à 2007 et par le Conseil de recherche en sciences humaines du Canada de 2007 à 2009. Remerciements chaleureux aux enseignants qui nous ont généreusement ouvert les portes de leur classe.

${ }^{3}$ Le lecteur intéressé à connaitre les résultats de la recherche dont les aspects conceptuels et méthodologiques sont discutés dans cet article pourra consulter Gauvin (2011; soumis) et Gauvin et Boivin (2012, soumis).

${ }^{4}$ Plusieurs savoirs sont impliqués dans l'accord du verbe, par exemple l'identification du verbe et du sujet.

${ }^{5}$ L'accord du verbe est défini comme le transfert des traits de nombre et de personne du noyau du GN (donneur) sujet au verbe (receveur). Le lecteur intéressé à approfondir l'aspect grammatical de la recherche consultera avec profit Gauvin (2011).

${ }^{6}$ Les transformations pourraient également être observées à partir des traces écrites des élèves lors de divers exercices décontextualisés ou lors d'activités de production écrite; nous n’avons pas retenu ces aspects pour notre recherche.

${ }^{7}$ La citation est reproduite telle quelle: les italiques et les guillemets sont de Chevellard (1985/1991).

${ }^{8}$ Suivant Boivin et Pinsonneault (2008), nous appelons manipulations décisives des manipulations syntaxiques qui identifient exclusivement une catégorie syntaxique ou une fonction grammaticale.

${ }^{9}$ L’astérisque indique que les phrases sont agrammaticales.

\section{Références des auteurs:}

Isabelle Gauvin est professeure au département de didactique des langues de l'UQAM depuis juin 2011. Elle s'intéresse à l'enseignement et à l'apprentissage de la grammaire, à l'utilisation par les élèves du secondaire de leurs connaissances grammaticales en écriture et à la formation des enseignants à la didactique de la grammaire et de l'écriture.

Marie-Claude Boivin est professeure agrégée au département de didactique de l’Université de Montréal. Elle est titulaire d'un doctorat en linguistique du Massachusetts Institute of Technology. Ses travaux portent sur la didactique de la grammaire (transposition, savoirs des élèves) et sur l'articulation de l'enseignement de la grammaire et de l'écriture.

Email: gauvin.isabelle@uqam.ca; marie-claude.boivin@umontreal.ca

Pour citer cet article:

Gauvin, I. \& Boivin, M.-C. (2012). La théorie de la transposition didactique: un outil conceptuel pour décrire les savoirs grammaticaux élaborés en classe par les élèves. Bellaterra Journal of Teaching \& Learning Language \& Literature, 5(3), 10-24. 